\title{
Substance abuse treatment outcomes in women with Fetal Alcohol Spectrum Disorder
}

\author{
Therese M. Grant ${ }^{1}$, Natalie Novick Brown ${ }^{1}$, J. Christopher Graham ${ }^{2}$, and Cara C. Ernst ${ }^{2}$ \\ ${ }^{1}$ University of Washington School of Medicine, Department of Psychiatry and Behavioral Sciences, Seattle, WA, United States \\ ${ }^{2}$ University of Washington Fetal Alcohol and Drug Unit, Alcohol and Drug Abuse Institute, Seattle, WA, United States
}

\begin{abstract}
Aims: There is little literature describing substance use treatment participation or completion rates for adults with neurocognitive impairments, including Fetal Alcohol Spectrum Disorders (FASD). This paper examines inpatient and outpatient substance abuse treatment outcomes among women with diagnosed or suspected FASD compared to women without prenatal alcohol exposure (PAE).
\end{abstract}

Design: Three-group observational study.

Setting: A three-year case management intervention program in Washington State for high-risk women who abuse alcohol and/or drugs during pregnancy.

Participants: Group 1: No PAE $(N=463)$; Group 2: Diagnosed with FASD (FAS, ARND, fetal alcohol effects, or static encephalopathy) by a qualified physician ( $N=25$; 14 included in hypothesis testing); Group 3: Suspected FASD (all reported PAE and displayed behaviors consistent with a clinical diagnosis of FASD) $(N=61)$.

Measures: Addiction Severity Index and biannual assessment of substance abuse treatment received and alcohol and drug use.

Findings: Women with diagnosed/suspected FASD were less likely than those without PAE to attend and complete inpatient and outpatient treatment. Women with diagnosed/suspected FASD who completed treatment were twice as likely to do so within a structured residential setting compared to a less structured outpatient setting.

Conclusions: Program structure in substance abuse treatment is important for people with FASD because of brain-based impairments. Outpatient treatment participation requires intact executive function skills (e.g., planning, organizing, sequencing), while structured inpatient treatment is not as challenging because it requires less independent decision-making. We suggest routine intake screening, referral protocols for follow-up diagnostic assessment, maximizing program structure to the extent possible, and modified therapeutic approaches to accommodate disabilities.

Prenatal alcohol exposure (PAE) may cause structural brain damage and central nervous system (CNS) abnormalities that contribute to psychosocial problems at every developmental stage (Autti-Rämö, 2000; Famy, Streissguth, \& Unis, 1998; Mattson \& Riley, 1998, 2000; Steinhausen \& Spohr, 1998; Thomas, Kelly, Mattson, \& Riley, 1998). Fetal Alcohol Spectrum Disorder (FASD) is an umbrella term for medical conditions involving CNS deficits caused by PAE, including Fetal Alcohol Syndrome (FAS), Partial FAS, and Alcohol-Related Neurodevelopmental Disorder (ARND). Individuals with FASD characteristically demonstrate impaired executive functioning, with associated difficulties in organizing stored information; planning future activities; regulating and sequencing behavior; making good judgments; foreseeing consequences and linking cause and effect; understanding abstract concepts; and thinking flexibly when faced with unexpected circumstances (Bookstein, Streissguth, Sampson, Connor, \& Barr, 2002; Connor, Sampson, Bookstein, Barr, \& Streissguth, 2000; Connor, Streissguth, Sampson, Bookstein, \& Barr, 1999; Kodituwakku, Handmaker, Cutler, Weathersby, \& Handmaker, 1995; Mattson, Goodman, Caine, Delis, \& Riley, 1999). All of these skills are critical for appropriate decision-making and day-to-day functioning, as even "mild" deficiencies (i.e., deficits that fall 1-1.5 standard deviations below the mean) can have a serious impact on an affected individual's life course (Streissguth, Bookstein, Barr, Sampson, O’Malley, \& Young, 2004), including decision-making about alcohol and drug use.

Correspondence: Therese Grant, Ph.D., University of Washington, 180 Nickerson St., Suite 309, Seattle, WA 98109, United States. Telephone: +1 206 543 7155; Fax: +1 206685 2903; E-mail: granttm@u.washington.edu

Financial support: Supported by the Washington State Department of Social and Health Services Division of Behavioral Health and Recovery, contracts $0965-69005$ and 1165-29940.

Keywords: fetal alcohol syndrome, fetal alcohol spectrum disorders, substance abuse treatment, inpatient, outpatient 
In their 1992-1996 evaluation of 415 patients with either FAS or fetal alcohol effects (FAE), Streissguth et al. (1996; 2004) found that $45.6 \%$ of the adults (age 21 and over) had a substance abuse problem, defined as having ever had alcohol or drug abuse problems or having ever received inpatient or outpatient substance abuse treatment (SAT). In contrast, results from the 1992 National Epidemiologic Survey on Alcohol and Related Conditions (NESARC) found an adult lifetime prevalence rate of $18.2 \%$ for alcohol use disorders in the U.S. (Vergés, Littlefield, \& Sher, 2011) and $10.3 \%$ for drug use disorders (Compton, Thomas, Stinson, \& Grant, 2007). Given that there are consistent, positive associations between substance use disorders and independent mood and anxiety disorders (Grant et al., 2004; Wolitzky-Taylor, Operskalski, Ries, Craske, \& RoyByrne, 2011), it may be that the propensity for individuals with FASD to abuse alcohol and drugs stems in large part from a relatively high degree of mood and anxiety disorders in this population (Brown, O’Malley, \& Streissguth, 2012). For example, a nation-wide prevalence study in 2001-2002 found mood disorders in 9.21\% and anxiety disorders in $11.08 \%$ of the adult population (Grant et al., 2004), whereas, in a sample of adults with FASD, rates of major depressive disorder (44\%), bipolar disorder (20\%), and anxiety disorders (20\%) were much higher (Famy et al., 1998).

Among people in the general population aged 12 and over who receive SAT, completion rates are low, particularly among clients in outpatient treatment. For example, among females discharged from outpatient treatment in 2005, 32\% completed treatment, $29 \%$ dropped out, and $11 \%$ were terminated by the facility (Substance Abuse and Mental Health Services Administration [SAMHSA], Office of Applied Studies [OAS], 2009a). In contrast, among females discharged from short-term inpatient treatment in 2005, 52\% completed treatment; among those discharged from long-term inpatient treatment, 38\% completed treatment (SAMHSA, OAS, 2009b). We suggest that this substantial treatment failure in the general population may be due in part to unidentified neurocognitive impairments such as FASD, which cause coping problems and other self-regulation difficulties during treatment (e.g., failing to follow treatment and facility rules).

There is little literature describing substance use treatment and interventions for adults with neurocognitive impairments. For instance, Burgard, Donohue, Azrin, and Geichner (2000) included no published SAT outcome studies in their review of studies examining alcohol and illegal drug use in individuals with mental retardation. We also found no information in the literature about treatment participation or completion rates for people with FASD. Consequently, the purpose of this paper is to examine this neglected aspect of treatment outcome research, specifically addressing outcomes among substance-abusing women with PAE who are enrolled in a three-year case management intervention. We hypothesize that compared to women without PAE, women with diagnosed or suspected FASD will have poorer SAT outcomes. We also hypothesize that environmental factors such as treatment setting will affect treatment completion.

\section{Methods}

\section{Participants}

The data presented in this study are from 549 women enrolled in the Washington State Parent-Child Assistance Program (PCAP) from November 1997 through July 2011. The PCAP model has been described elsewhere (Ernst, Grant, Streissguth, \& Sampson, 1999; Grant, Ernst, Pagalilauan, \& Streissguth, 2003; Grant, Ernst, Streissguth, \& Stark, 2005). In brief, the program is a three-year home visitation and case management intervention for high-risk women who abuse alcohol and/or drugs during pregnancy. The primary aims of PCAP are to assist women in obtaining treatment, maintaining recovery, and resolving problems associated with substance abuse. PCAP case managers (CM) are paraprofessionals with a 16-family caseload who conduct home visits approximately twice per month and connect women and their families with community services. CMs are supervised by experienced clinicians highly trained in FASD and credentialed in the mental health, social work, or chemical dependency fields. Standard PCAP eligibility criteria include (1) currently pregnant or up to six months postpartum; (2) self-reported heavy alcohol and/or illicit drug use during the index pregnancy; and (3) ineffective or nonexistent engagement with community services. Participants in PCAP are referred by social service providers (e.g., public health nurses).

For the present study, we categorized cases into three groups:

Group 1: No PAE ( $N=463)$. Women in Group 1 were enrolled under standard PCAP eligibility criteria. None reported having a mother who drank alcohol during pregnancy with the client, and all completed the three-year PCAP intervention.

Group 2: Diagnosed with an FASD $(N=25)$. In 1999, PCAP expanded eligibility to enroll women who had been diagnosed with an FASD (including FAS, ARND, fetal alcohol effects, or static encephalopathy) at some time prior to being referred to PCAP. Twenty-five women were enrolled under this criterion. All had been diagnosed by a qualified physician or FASD multidisciplinary diagnostic team, which was verified by clinic records. Their average age at time of FASD diagnosis was 16 years (range: birth to 45 years); 21 had children or were pregnant at intake, and four had no children. Although they were not required to meet PCAP enrollment criteria, 16 (64\%) were enrolled during pregnancy or postpartum. While most (24 of 25) had a history of substance use, only 14 (56\%) reported having a substance abuse problem, defined as either (1) self-reported methamphetamine, cocaine, heroin, or binge alcohol use during the index pregnancy or during the 30 days prior to intake, or (2) self-reported prior inpatient/outpatient SAT. All women in Group 2 completed the three-year PCAP intervention.

Group 3: Suspected FASD $(N=\mathbf{6 1})$. Women in this group were enrolled under standard PCAP eligibility criteria. PAE was self-reported during intake $(65.9 \%$ answered yes to the query, "Did your mother drink alcohol when she was pregnant with you?”), or was subsequently 
disclosed to the CM by the client, client's mother, or another family member. On the intake interview, women in Group 3 reported histories of learning and social problems across the lifespan, and during PCAP the customary adult behaviors they displayed (while not under the influence of alcohol or drugs) were consistent with a clinical diagnosis of FASD. These observations were documented by PCAP clinical supervisors, and all notes were reviewed by co-authors TG and CE. All 61 women in Group 3 are currently enrolled in PCAP.

Institutional Review Board approval was obtained from the Washington State Institutional Review Board, and a certificate of confidentiality was obtained from the federal Department of Health and Human Services.

\section{Measures}

Addiction Severity Index. At intake and three-year program exit, all participants were interviewed using the Addiction Severity Index (ASI) (McLellan et al., 1992), an instrument widely used for clinical assessment and research purposes. In 1997, PCAP researchers developed supplemental questions regarding childhood history, community services, family planning, and substance use during pregnancy.

Biannual assessment. Every six months throughout the intervention, CMs completed a checklist assessment of client status, including SAT received and alcohol and drug use. In an earlier report (Grant et al., 2011), we compared clients' ASI reports of past 30-day substance use at program exit with the CM checklist response on the 36month assessment. In only $5.8 \%$ of the cases did the CM report client substance use when the client had not reported it.

Dependent variables. "Any inpatient treatment” was defined as involvement in short-term (i.e., up to 30 days) or long-term (i.e., up to 180 days) residential treatment. "Any outpatient treatment" was defined as involvement in at least one of the following: (1) non-intensive outpatient program treatment, recovery, or aftercare services; (2) non-intensive treatment services provided by individual outpatient providers, including counseling and supportive services; and (3) intensive outpatient services involving three or more hours per day for at least three days per week.

\section{Data Analyses}

We tested for overall group differences on continuous variables using ANOVA, followed by post-hoc tests (Least Significant Difference) if significant overall. For categorical variables, we used Fisher's Exact Test for 2x2 tables and Pearson's Chi-Square for tables that included more categories. Tests with results reported in Table 1 are two-tailed tests, while tests reported in Table 2 are onetailed tests (consistent with the directional hypothesis).

For analyses of cumulative treatment outcomes 24 months after PCAP intake (results in Table 2), we compared Group 1 with a group composed of all women in Group 3 (as all had substance abuse problems) plus the 14 women in Group 2 who had substance abuse problems. Our cumulative treatment hierarchy was defined as follows:
1 = Completed (ever); 2 = In Progress (in progress at 24 months, or earlier if all subsequent measurements are missing); 3 = Dropped (stopped treatment at any point during the intervention).

For informational purposes only, Table 1 includes a column presenting descriptive data for the subgroup of 14 women in Group 2 who were used for the primary analysis. Similarly, Table 2 includes two columns presenting data for the women in Groups 2 and 3 who compose the combined analysis group.

\section{Results}

\section{Intake maternal demographic and psychosocial characteristics (Table 1)}

Regardless of group, the majority of women in this study had traumatic or atypical life histories. For example, most were sexually abused as children (53.4\% to $60.0 \%)$ and had histories involving incarceration (60.0\% to $77.0 \%)$. A sizable number had been physically abused in childhood (38.6\% to $57.4 \%$ ). Many had been raised by caregivers other than their birth parents (26.4\% to $76.0 \%)$.

Group 2 (FASD). At intake, women in Group 2 (FASD) were younger than women in Group 1 (no PAE/FASD) (mean $=23.2$ vs. 26.8 years, $p<.01$ ) and had fewer children compared to women in Groups 1 and 3 (means $=2.7,1.7,2.8$, respectively, $p<.01$ ). Compared to the other two groups, a higher proportion of Group 2 women lived in permanent or stable housing (34.6\%, $56.0 \%, 29.5 \%$, respectively, $p<.01$ ), and had never held a full-time job $(24.0 \%, 64.0 \%, 32.8 \%, p<.001)$.

Group 3 (suspected FASD). Compared to women in Group 1, a significantly higher proportion of women in Group 3 had been physically abused as children (38.6\% vs. $57.4 \%, p<.05$ ), had no high school diploma (48.3\% vs. $63.9 \%, p<.05)$, and were homeless or living in temporary quarters at PCAP intake (30.5\% vs. $47.5 \%, p<.01)$.

Groups 2 and 3 (FASD/suspected FASD) vs. Group 1 (no PAE/FASD). Compared to women in Group 1, greater proportions of women in Groups 2 and 3 had not lived with their birth parent(s) throughout childhood (26.4\%, 76.0\%, $59.0 \%, p<.001)$ and had significantly fewer years of education $($ mean $=11.1,10.2,10.5, p<.01)$.

Psychiatric symptomatology. Across all groups, over $60 \%$ of women reported having psychiatric problems in the preceding 30 days, and significantly more women in Group $3(80.0 \%)$ compared to Group $1(62.8 \%)$ reported such problems $(p<.05)$. Compared to women in Group 1, Groups 2 and 3 reported similar high rates of trouble concentrating $(42.3 \%, 79.2 \%, 67.2 \%, p<.001)$. Lifetime suicide attempts were high, with significant differences between Group 1 and Group 3 (31.7\% vs. 47.5\%, $p<.05$ ). With respect to mood disorders in particular, women in Group 3 were approximately twice as likely to report symptoms of depression $(37.2 \%, 37.5 \%, 70.5 \%, p<.001)$ and anxiety symptoms $(42.4 \%, 37.5 \%, 72.1 \%, p<.001)$ as women in Groups 1 and 2, and much more likely to report violent behavior $(4.5 \%, 8.3 \%, 19.7 \%, p<.001)$. 
Table 1

Maternal demographic and psychosocial characteristics at program intake

\begin{tabular}{|c|c|c|c|c|c|}
\hline Characteristic & $\begin{array}{c}\text { Group } 1 \text { No PAE } \\
\text { or FASD } \\
(N=463)^{1} \\
\end{array}$ & $\begin{array}{c}\text { Group } 2 \text { FASD } \\
\text { Diagnosis } \\
(N=25)^{2} \\
\end{array}$ & $\begin{array}{c}\text { Group } 3 \text { FASD } \\
\text { Suspected } \\
(N=61)^{3}\end{array}$ & $p$ & $\begin{array}{c}\text { Group } 2 \text { (Used } \\
\text { in Analysis) } \\
(N=14)^{5}\end{array}$ \\
\hline \multicolumn{6}{|l|}{ Maternal Age } \\
\hline Mean years & $26.8^{2,3}$ & $23.2^{1}$ & $24.9^{1}$ & ** & 24.6 \\
\hline Range & $15-45$ & $14-42$ & $19-39$ & & $15-42$ \\
\hline$S D$ & 6.2 & 6.1 & 5.0 & & 6.5 \\
\hline \multicolumn{6}{|l|}{ Parity: Total \# children } \\
\hline Mean & $2.7^{2}$ & $1.7^{1,3}$ & $2.8^{2}$ & $* *$ & 2.1 \\
\hline Range & $1-9$ & $0-6$ & $1-9$ & & $0-6$ \\
\hline$S D$ & 1.6 & 1.3 & 1.9 & & 1.4 \\
\hline \multicolumn{6}{|l|}{ Race } \\
\hline White & $296(63.9 \%)^{+}$ & $14(56.0 \%)$ & $31(50.8 \%)$ & 6 & $6(42.9 \%)$ \\
\hline Native American & $60(13.0 \%)^{-}$ & $7(28.0 \%)$ & $18(29.5 \%)^{+}$ & & $6(42.9 \%)$ \\
\hline Asian / Black / Hispanic & 107 (23.1\%) & $4(16.0 \%)$ & $12(19.7 \%)$ & & $2(14.3 \%)$ \\
\hline \multicolumn{6}{|l|}{ Marital } \\
\hline Never married & $318(68.7 \%)$ & $20(80.0 \%)$ & $43(70.5 \%)$ & 6 & $12(85.7 \%)$ \\
\hline \multicolumn{6}{|l|}{ Main Income Source } \\
\hline Welfare & $296(63.9 \%)$ & 9 (36.0\%)- & $37(60.7 \%)$ & 6 & $7(50.0 \%)$ \\
\hline Family / Friends / Other & $118(25.5 \%)$ & $8(32.0 \%)$ & $16(26.2 \%)$ & & $4(28.6 \%)$ \\
\hline SSI, Tribal & $35(7.6 \%)^{-}$ & $7(28.0 \%)^{+}$ & $7(11.5 \%)$ & & $3(21.4 \%)$ \\
\hline \multicolumn{6}{|l|}{ Housing } \\
\hline Permanent/Stable housing & 160 (34.6\%) & $14(56.0 \%)^{+}$ & $18(29.5 \%)$ & $* *$ & $7(50.0 \%)$ \\
\hline $\begin{array}{l}\text { Substance abuse treatment or drug-free } \\
\text { transitional housing }\end{array}$ & $161(34.8 \%)^{+}$ & $2(8.0 \%)^{-}$ & $14(23.0 \%)$ & & $1(7.1 \%)$ \\
\hline Temporary or homeless & $141(30.5 \%)^{-}$ & $9(36.0 \%)$ & $29(47.5 \%)^{+}$ & & $6(42.9 \%)$ \\
\hline \multicolumn{6}{|l|}{ Education } \\
\hline No high school diploma & $223(48.3 \%)^{-}$ & $17(68.0 \%)$ & $39(63.9 \%)^{+}$ & $*$ & $8(57.1 \%)$ \\
\hline Mean years & $11.1^{2,3}$ & $10.2^{1}$ & $10.5^{1}$ & $* *$ & 10.7 \\
\hline Range & $4-16$ & 6-13 & $7-12$ & & $7-13$ \\
\hline$S D$ & 1.9 & 1.9 & 1.5 & & 1.9 \\
\hline \multicolumn{6}{|l|}{ Employment } \\
\hline \multicolumn{6}{|l|}{ If ever a FT job: } \\
\hline Mean years & 2.4 & 1.7 & 1.6 & ns & 2.1 \\
\hline Range & $0.08-20$ & $0.17-7$ & $0.08-13$ & & $0.33-7$ \\
\hline$S D$ & 2.5 & 2.1 & 2.3 & & 2.3 \\
\hline \multicolumn{6}{|l|}{ Childhood trauma } \\
\hline Physically abused & $178(38.6 \%)^{-}$ & $11(44.0 \%)$ & $35(57.4 \%)^{+}$ & $*$ & $5(35.7 \%)$ \\
\hline Sexually abused & $243(53.4 \%)$ & $15(60.0 \%)$ & 34 (56.7\%) & ns & $6(42.9 \%)$ \\
\hline Did not always live with birth parents & $122(26.4 \%)^{-}$ & $19(76.0 \%)^{+}$ & $36(59.0 \%)^{+}$ & $* * *$ & $11(78.6 \%)$ \\
\hline \multicolumn{6}{|l|}{ Criminal justice involvement } \\
\hline Ever incarcerated & $352(76.4 \%)$ & $15(60.0 \%)$ & $47(77.0 \%)$ & $n s$ & $10(71.4 \%)$ \\
\hline Mean \# times & $5.2^{2,3}$ & $13.6^{1,3}$ & $7.8^{12}$ & $* * *$ & 10.5 \\
\hline Range & $0-98$ & $0-40$ & $0-108$ & & $0-40$ \\
\hline$S D$ & 10.2 & 12.7 & 16.3 & & 14.7 \\
\hline \multicolumn{6}{|l|}{ Psychiatric symptoms, past 30 days } \\
\hline Any problems reported & $290(62.8 \%)^{-}$ & $19(79.2 \%)$ & $48(80.0 \%)^{+}$ & $*$ & $11(78.6 \%)$ \\
\hline Depression & $172(37.2 \%)^{-}$ & $9(37.5 \%)$ & $43(70.5 \%)^{+}$ & $* * *$ & $8(57.1 \%)$ \\
\hline Anxiety & $196(42.4 \%)^{-}$ & $9(37.5 \%)$ & $44(72.1 \%)^{+}$ & $* * *$ & $7(50 \%)$ \\
\hline Trouble concentrating & $195(42.3 \%)^{-}$ & $19(79.2 \%)^{+}$ & $41(67.2 \%)^{+}$ & $* * *$ & $10(71.4 \%)$ \\
\hline Violent behavior & $21(4.5 \%)^{-}$ & $2(8.3 \%)$ & $12(19.7 \%)^{+}$ & $* * *$ & $0(0.0 \%)$ \\
\hline Ever a suicide attempt & $147(31.7 \%)^{-}$ & $10(41.7 \%)$ & $29(47.5 \%)^{+}$ & $*$ & $6(42.9 \%)$ \\
\hline
\end{tabular}

${ }^{1,2,3}$ Groups with means that are significantly different $(p<.05)$ are indicated by a superscript reflecting the group(s) from which they are different.

${ }^{4}$ Statistical tests are based upon the groups represented to the left (particular variables may have some missing values). Groups with categorical percentages significantly greater or less than what would be expected from the marginal distributions are indicated by valence superscripts (+ or - ).

${ }^{5}$ This column presents information for the subgroup of women in Group 2 (FASD Diagnosis) included in the study's main analysis (results presented in Table 2).

${ }^{6}$ Test not warranted because one or more cells had expected value of less than 5 .

${ }^{*} p<.05, * * p<.01, * * * p<.001$ 


\section{Cumulative treatment outcomes at $\mathbf{2 4}$ months for outpatient and inpatient service settings (Table 2)}

Any outpatient treatment. After being in PCAP for 24 months, significantly fewer women in the Combined FASD Group (PAE and diagnosed or suspected FASD) had attended outpatient treatment compared to women in Group 1 (no PAE or FASD) (79.2\% vs. 89.8\%, $p<.05$ ). Those with PAE appeared to have lower completion rates than those without PAE $(29.8 \%$ vs. $42.9 \%, p=.07$, marginal), though similar proportions in both groups were still in progress with outpatient treatment $(49.1 \%$ vs. $42.7 \%, p=.07$, marginal). A higher proportion of women with PAE dropped out of outpatient treatment (21.1\% vs. $14.5 \%, n s)$.

Any inpatient treatment. We observed a similar trend for inpatient residential treatment. That is, after 24 months it appeared that fewer women in the Combined FASD Group had received any inpatient treatment compared to women in Group 1 (45.8\% vs. 55.4\%, $p=.08$, marginal). However, among women who received inpatient treatment, those with or without PAE were nearly equally likely (63.6\% and $69.5 \%$ respectively) to complete treatment. A somewhat higher proportion of women with PAE dropped out of treatment (27.3\% vs. 19.1\%), though the extreme marginal distributions precluded significance testing.

Any inpatient/outpatient treatment. Comparing the two groups in terms of treatment completion regardless of treatment modality, significantly fewer women in the Combined FASD Group participated in any kind of treatment (83.3\% vs. $92.0 \%, p<.05)$, and, of those who did participate, significantly more women in the Combined FASD Group dropped out $(21.7 \%$ vs. 9.6\% $p<.01)$.

\section{Abstinence from alcohol and drugs at PCAP 3- year exit (data not shown in table)}

We also examined alcohol and drug abstinence outcomes between two groups of women who had completed the three-year intervention: Group $1(N=462)$ and members of Group 2 who had a substance abuse problem $(N=13$; one had missing data). Three abstinence outcomes were examined: (1) abstinence for six months or longer at PCAP exit (45.7\% vs. $46.2 \%$, respectively, $n s$ ); (2) abstinence for 12 months or longer at PCAP exit (39.0\% vs. 30.8\%, ns); and (3) abstinence for 12 months or longer sometime during the PCAP intervention (64.0\% vs. $46.2 \%, n s)$. None of the differences was statistically significant, because of small cell sizes in Group 2.

Table 2

Cumulative SAT outcomes at 24 months by outpatient and inpatient service setting

\begin{tabular}{|c|c|c|c|c|c|}
\hline Characteristic & $\begin{array}{c}\text { Group } 1 \\
\text { No PAE or FASD } \\
(N=462)^{1}\end{array}$ & $\begin{array}{l}\text { Combined FASD } \\
\text { Group (Diagnosed } \\
\text { or Suspected } \\
\text { FASD) } \\
(N=72)^{2} \\
\end{array}$ & $p$ & $\begin{array}{c}\text { Group } 2 \text { FASD } \\
\text { Diagnosis (Used } \\
\text { in Analysis) } \\
(N=14)^{4}\end{array}$ & $\begin{array}{c}\text { Group } 3 \text { FASD } \\
\text { Suspected (Used } \\
\text { in Analysis) } \\
(N=14)^{2,5}\end{array}$ \\
\hline \multicolumn{6}{|l|}{ At 24 months: } \\
\hline $\begin{array}{l}\text { Any Outpatient Treatment } \\
\text { Status }\end{array}$ & $415(89.8 \%)^{+}$ & $57(79.2 \%)^{-}$ & $*$ & $10(71.4 \%)$ & 47 (81.0\%) \\
\hline Completed & $178(42.9 \%)$ & 17 (29.8\%) & ns & $6(60.0 \%)$ & $11(23.4 \%)$ \\
\hline In progress & $177(42.7 \%)$ & $28(49.1 \%)$ & & $2(20.0 \%)$ & $26(55.3 \%)$ \\
\hline Dropped & $60(14.5 \%)$ & $12(21.1 \%)$ & & $2(20.0 \%)$ & $10(21.3 \%)$ \\
\hline $\begin{array}{l}\text { Any Inpatient Treatment } \\
\text { Status }\end{array}$ & $256(55.4 \%)$ & $33(45.8 \%)$ & $n s$ & $5(35.7 \%)$ & $28(48.3 \%)$ \\
\hline Completed & $178(69.5 \%)$ & $21(63.6 \%)$ & 6 & $2(40.0 \%)$ & $19(67.9 \%)$ \\
\hline In progress & 29 (11.3\%) & $3(9.1 \%)$ & & $2(40.0 \%)$ & $1(3.6 \%)$ \\
\hline Dropped & 49 (19.1\%) & $9(27.3 \%)$ & & $1(20.0 \%)$ & $8(28.6 \%)$ \\
\hline $\begin{array}{l}\text { Any In/Outpatient Treatment } \\
\text { Status }\end{array}$ & $425(92.0 \%)^{+}$ & $60(83.3 \%)^{-}$ & $*$ & $10(71.4 \%)$ & $50(86.2 \%)$ \\
\hline Completed & $270(63.5 \%)$ & $31(51.7 \%)$ & $* *$ & $7(70.0 \%)$ & $24(48.0 \%)$ \\
\hline In progress & $114(26.8 \%)$ & $16(26.7 \%)$ & & $2(20.0 \%)$ & $14(28.0 \%)$ \\
\hline Dropped & $41(9.6 \%)^{-}$ & $13(21.7 \%)^{+}$ & & $1(10.0 \%)$ & $12(24.0 \%)$ \\
\hline
\end{tabular}

${ }^{1}$ One woman in this group was missing all measures of treatment outcome status, and so could not be included in the analysis.

${ }^{2}$ Three women in the combined group (from Group 3) were missing all measures of treatment outcome status, and so could not be included in the analysis.

${ }^{3}$ Groups with categorical percentages significantly greater or less than what would be expected from the marginal distributions are indicated by valence superscripts ( + or - ). The statistical tests were made with reference to the groups presented to the left.

${ }^{4,5}$ These columns present information for the Group 2 and Group 3 members who compose the "Combined” analysis group to the left.

${ }^{6}$ Test not warranted because one or more cells had expected value of less than 5 .

${ }^{*} p<.05, * * p<.01, * * * p<.001$ 


\section{Discussion}

Results of this study support two important conclusions: both PAE and program structure matter in terms of successful treatment outcome for substance-abusing women. It is notable that women in our study with diagnosed or suspected FASD had an outpatient treatment completion rate $(29.8 \%)$ similar to rates in the general female population (32\%), and their inpatient treatment completion rate $(63.6 \%)$ was higher than rates in the general female population (38\% to $52 \%$ depending on length of stay) (SAMHSA, OAS, 2009a, 2009b). However, compared to women in our study without PAE/FASD who also received intensive three-year case management and PCAP support, women with diagnosed or suspected FASD were less likely to participate in inpatient or outpatient SAT, and among those who did participate, those with FASD/suspected FASD were less likely to complete such treatment. Furthermore, on two of the three abstinence measures assessed at PCAP exit, women with PAE were less likely to abstain from alcohol and drugs than women without PAE. These findings are consistent with our hypothesis.

Program structure appears to be important. Similar to what has been observed in the general population, our study found higher completion rates for inpatient treatment compared to outpatient treatment, regardless of PAE status. However, among women with PAE, over twice as many completed treatment within residential settings compared to outpatient settings. We postulate that the reason program setting is so important in SAT outcomes is because the increased program structure of inpatient settings may compensate for brain-based impairments in FASD. That is, participating regularly in outpatient treatment requires intact executive functioning skills (e.g., prioritizing, planning daily activities around treatment sessions, coping with unforeseen events, arranging for transportation). In contrast, structured inpatient treatment is less challenging in this regard, because daily planning and organization are the responsibility of staff, which reduces the need for clients' independent decision-making and self-regulation.

Demographic findings in this study are consistent with previous FASD research (Greenbaum, Stevens, Nash, Koren, \& Rovet, 2009) in that women with PAE appear to experience more difficult life circumstances in general than women without PAE (e.g., not living with birth parent[s] throughout childhood, lower education level, increased psychiatric symptomatology). In addition, this study replicates the secondary disability literature in finding that diagnostic status is an important consideration in terms of life-course outcomes. As adults, undiagnosed women with PAE tended to have unstable housing, higher rates of criminal justice involvement, and more psychiatric symptoms than those diagnosed with FASD. The timing of the diagnosis appears to be quite important, as Streissguth and colleagues (2004) found that one of the strongest correlates of adverse life outcomes among people with FASD was lack of an early childhood diagnosis; the earlier the diagnosis, the greater the opportunity for capable caregivers to implement interventions and effectively advocate for their vulnerable family members' needs.

\section{Limitations}

Study intake and exit data were obtained from maternal interviews and therefore were subject to possible self-report bias (Rothman, 1986). We did not collect data about whether treatment programming was geared specifically toward women. In spite of apparent trends observed in outcomes reported, many findings did not reach statistical significance, or could not be tested, because of small cell sample sizes. Moreover, since childhood environmental adversity appears to be more prevalent among individuals with FASD than those without FASD (Greenbaum et al., 2009), future treatment outcome studies should account for such history.

Unfortunately, because most SAT programs do not assess patients for possible brain damage on a routine basis or individualize treatment planning for patients diagnosed with neurocognitive impairments, patients with PAE are left to fend for themselves. It is not surprising then that such individuals have considerable difficulty completing treatment unless they receive substantial support. Beyond the importance of providing as much structure within the program as is possible, we suggest that SAT for adults include routine screening at intake, referral protocols regarding follow-up diagnostic assessment, modified treatment curricula to accommodate specific disabilities that affect treatment progress, and intensified aftercare support.

\section{Acknowledgments}

We extend special thanks to the Parent-Child Assistance Program (PCAP) case managers and clinical supervisors, and to the women enrolled in the program for their valuable contributions to this work. This work was supported by the State of Washington Department of Social and Health Services (DSHS) contracts \#0965-69005 and \#1165-29940.

\section{References}

Autti-Rämö, I. (2000). Twelve-year follow-up of children exposed to alcohol in utero. Developmental Medicine \& Child Neurology, 42, 406-411.

Bookstein, F. L., Streissguth, A. P., Sampson, P. D., Connor, P. D., \& Barr, H. M. (2002). Corpus callosum shape and neuropsychological deficits in adult males with heavy fetal alcohol exposure. NeuroImage, 15, 233-251.

Brown, N. N., O’Malley, K., \& Streissguth, A. P. (2012). FASD: Diagnostic dilemmas and challenges for a modern transgenerational management approach. In S. Adubato \& D. Cohen (Eds.), Prenatal alcohol use and fetal alcohol spectrum disorders: Diagnosis, assessment, and new directions in research and multimodal treatment. Bentham Online Publishing. 
Burgard, J. F., Donohue, B., Azrin, N. H., \& Geichner, G. (2000). Prevalence and treatment of substance abuse in the mentally retarded population: An empirical review. Journal of Psychoactive Drugs, 32, 293-298.

Compton, W. M., Thomas, Y. F., Stinson, F. S., \& Grant, B. F. (2007). Prevalence, correlates, disability, and comorbidity of DSM-IV drug abuse and dependence in the United States: Results from the national epidemiologic survey on alcohol and related conditions. Archives of General Psychiatry, 64, 566576.

Connor, P. D., Sampson, P. D., Bookstein, F. L., Barr, H. M., \& Streissguth, A. P. (2000). Direct and indirect effects of prenatal alcohol damage on executive function. Developmental Neuropsychology, 18, 331354.

Connor, P. D., Streissguth, A. P., Sampson, P. D., Bookstein, F. L., \& Barr, H. M. (1999). Executive functioning deficits in adults prenatally exposed to alcohol. Alcoholism: Clinical and Experimental Research, 23, 1395-1402.

Ernst, C. C., Grant, T. M., Streissguth, A. P., \& Sampson, P. D. (1999). Intervention with high-risk alcohol and drug-abusing mothers: II. Three-year findings from the Seattle model of paraprofessional advocacy. Journal of Community Psychology, 27, 19-38.

Famy, C., Streissguth, A. P., \& Unis, A. S. (1998). Mental illness in adults with fetal alcohol syndrome or fetal alcohol effects. American Journal of Psychiatry, 155, 552-554.

Grant, B. F., Stinson, F. S., Dawson, D. A., Chou, S. P., Dufour, M. C., Compton, W., ... Kaplan, K. (2004). Prevalence and co-occurrence of substance use disorders and independent mood and anxiety disorders: results from the national epidemiologic survey on alcohol and related conditions. Archives of General Psychiatry, 61, 807-816.

Grant, T., Ernst, C. C., Pagalilauan, G., \& Streissguth, A. P. (2003). Post-program follow-up effects of paraprofessional intervention with high-risk women who abused alcohol and drugs during pregnancy. Journal of Community Psychology, 31, 211-222.

Grant, T., Ernst, C. C., Streissguth, A., \& Stark, K. (2005). Preventing alcohol and drug exposed births in Washington State: Intervention findings from three parent-child assistance program sites. American Journal of Drug and Alcohol Abuse, 31, 471-490.

Grant, T. M., Huggins, J., Graham, J. C., Ernst, C., Whitney, N., \& Wilson, D. (2011). Maternal substance abuse and disrupted parenting: Distinguishing mothers who keep their children from those who do not. Children and Youth Services Review, 33, 2176-2185.

Greenbaum, R. L., Stevens, S. A., Nash, K. Koren, G., \& Rovet, J. (2009). Social cognitive and emotion processing abilities of children with fetal alcohol spectrum disorders: A comparison with attention deficit hyperactivity disorder. Alcoholism: Clinical and Experimental Research, 33, 1656-1670.

Kodituwakku, P. W., Handmaker, N. S., Cutler, S. K., Weathersby, E. K., \& Handmaker, S. D. (1995). Specific impairments in self-regulation in children exposed to alcohol prenatally. Alcoholism: Clinical and Experimental Research, 19, 1558-1564.

Mattson, S. N., Goodman, A. M., Caine, C., Delis, D. C., \& Riley, E. P. (1999). Executive functioning in children with heavy prenatal alcohol exposure. Alcoholism: Clinical and Experimental Research, 23, 1808-1815.

Mattson, S. N. \& Riley, E. P. (1998). A review of the neurobehavioral deficits in children with fetal alcohol syndrome or prenatal exposure to alcohol. Alcoholism: Clinical and Experimental Research, 22, 279-294.

Mattson, S. N. \& Riley, E. P. (2000). Parent ratings of behavior in children with heavy prenatal alcohol exposure and IQ-matched controls. Alcoholism: Clinical and Experimental Research, 24, 226-231.

McLellan, A. T., Kushner, H., Metzger, D., Peters, R., Smith, I., Grissom, G., ... Argeriou, M. (1992). The fifth edition of the addiction severity index. Journal of SAT, 9, 199-213.

Rothman, K. J. (1986). Modern epidemiology. Boston/Toronto: Little Brown and Company.

Steinhausen, H. C. \& Spohr, H. L. (1998). Long-term outcome of children with fetal alcohol syndrome: psychopathology, behavior, and intelligence. Alcoholism: Clinical and Experimental Research, 22, 334-338

Streissguth, A., Barr, H., Kogan, J., \& Bookstein, F. (1996). Understanding the occurrence of secondary disabilities in clients with fetal alcohol syndrome (FAS) and fetal alcohol effects (FAE). Final report to the Centers for Disease Control and Prevention $(C D C)$. Seattle, WA, United States: University of Washington, Fetal Alcohol \& Drug Unit.

Streissguth, A. P., Bookstein, F. L., Barr, H. M., Sampson, P. D., O’Malley, K., \& Young, J. K. (2004). Risk factors for adverse life outcomes in fetal alcohol syndrome and fetal alcohol effects. Developmental and Behavioral Pediatrics, 25, 228-238.

Substance Abuse and Mental Health Services Administration(SAMHSA), Office of Applied Studies (OAS). (2009a). The TEDS report: Treatment outcomes among clients discharged from residential SAT: 2005. Rockville, MD., United States: Author.

Substance Abuse and Mental Health Services Administration(SAMHSA), Office of Applied Studies (OAS). (2009b). The TEDS report: Treatment outcomes among clients discharged from outpatient SAT. Rockville, MD., United States: Author.

Thomas, S. E., Kelly, S. J., Mattson, S. N., \& Riley, E. P. (1998). Comparison of social abilities of children with fetal alcohol syndrome to those of controls with children with similar IQ scores and normal controls. Alcoholism: Clinical and Experimental Research, 22, 528-533.

Vergés, A., Littlefield, A. K., \& Sher, K. J. (2011). Did lifetime rates of alcohol use disorders increase by $67 \%$ in 10 years? A comparison of NLAES and NESARC. Journal of Abnormal Psychology, 120, 868-877.

Wolitzky-Taylor, K., Operskalski, J. T., Ries, R., Craske, M. G., \& Roy-Byrne, P. (2011). Understanding and treating comorbid anxiety disorders in substance users: Review and future directions. Journal of Addiction Medicine, 5, 233-247. 